\title{
Cosmic rays and new fermionic dark matters
}

\author{
Jae-Kwang Hwang ${ }^{1}$ \\ JJJ Physics Laboratory, Brentwood, TN 37027 USA \\ E-mail: jkhwang.koh@gmail.com
}

Three generations of leptons and quarks correspond to the lepton charges (LC) in the present work. Then, the leptons have the electric charges (EC) and lepton charges (LC). The quarks have the EC, LC and color charges (CC). Three heavy leptons and three heavy quarks are introduced to make the missing third flavor of EC. Then the three new particles which have the electric charges (EC) are proposed as the bastons (dark matters) with the rest masses of $26.121 \mathrm{eV} / \mathrm{c}^{2}, 42.7$ $\mathrm{GeV} / \mathrm{c}^{2}$ and $1.910^{15} \mathrm{eV} / \mathrm{c}^{2}$. These new particles are applied to explain the origins of the astrophysical observations like the ultra-high energy cosmic rays and super-nova 1987A anti neutrino data. It is concluded that the $3.5 \mathrm{keV} x$ ray peak observed from the cosmic $\mathrm{x}$-ray background spectra is originated not from the pair annihilations of the dark matters but from the $\mathrm{x}$-ray emission of the Q1 baryon atoms which are similar in the atomic structure to the hydrogen atom. New particles can be indirectly seen from the astrophysical observations like the cosmic rays and cosmic gamma rays.

35th International Cosmic Ray Conference - ICRC2017-

10-20 July, 2017

Bexco, Busan, Korea

\section{${ }^{1}$ Speaker}




\section{Introduction}

The new physics search beyond standard model has been done by the extended standard models with the new particles. These new particles include the SUSY particles, techniquarks, leptoquarks, Z-prime boson, W-prime boson and heavy quarks (T, B, X and Y), sterile $v$, neutralinos, $\mathrm{X}$ - and Y- bosons, WIMPS, axions, preons. But there are no experimental evidences for these new particles. The previously known models including the string theory and the supersymmetry model have been developed on the unquantized space. In the present work, the three-dimensional quantized space model is introduced as the new extended standard model for the new elementary particles in Tables 1 and 2. The three dark matters (B1,B2,B3), three heavy leptons $(\mathrm{Le}, \mathrm{L} \mu, \mathrm{L} \tau)$ and three heavy quarks $(\mathrm{Q} 1, \mathrm{Q} 2, \mathrm{Q} 3)$ are introduced. The rest masses of the quarks, leptons and dark matters are calculated by using the simple equations to show the energy scales. These new particles can be indirectly searched for from the astrophysical observations like the cosmic rays and cosmic gamma rays. The unsolved questions of the astrophysical observations are explained by using the decays and interactions of these new particles in the present work. For example, the ultra-high energy cosmic rays are proposed to be originated from the decays and annihilations of the hadrons including the new heavy quarks (Q1,Q2,Q3). The super-nova 1987A is discussed in the relation with the B1 dark matter. And it is concluded that the observed 18.7 $\mathrm{keV}, 3.5 \mathrm{keV}$ and $74.9 \mathrm{keV}$ x-ray peaks are emitted from the Q1 baryon atoms. The rest masses of the B1 and B2 dark matters are proposed as $26.121 \mathrm{eV} / \mathrm{c}^{2}$ and $42.7 \mathrm{GeV} / \mathrm{c}^{2}$, respectively. The dark matter core collapse is newly introduced in addition to the normal matter core collapse to explain the super-nova explosion.

\section{Three-dimensional quantized space model and fermionic dark matters}

In Table 1, the leptons and quarks have the same properties of the three generations. Three generations separate the leptons and quarks with the same electric charges (EC). Three

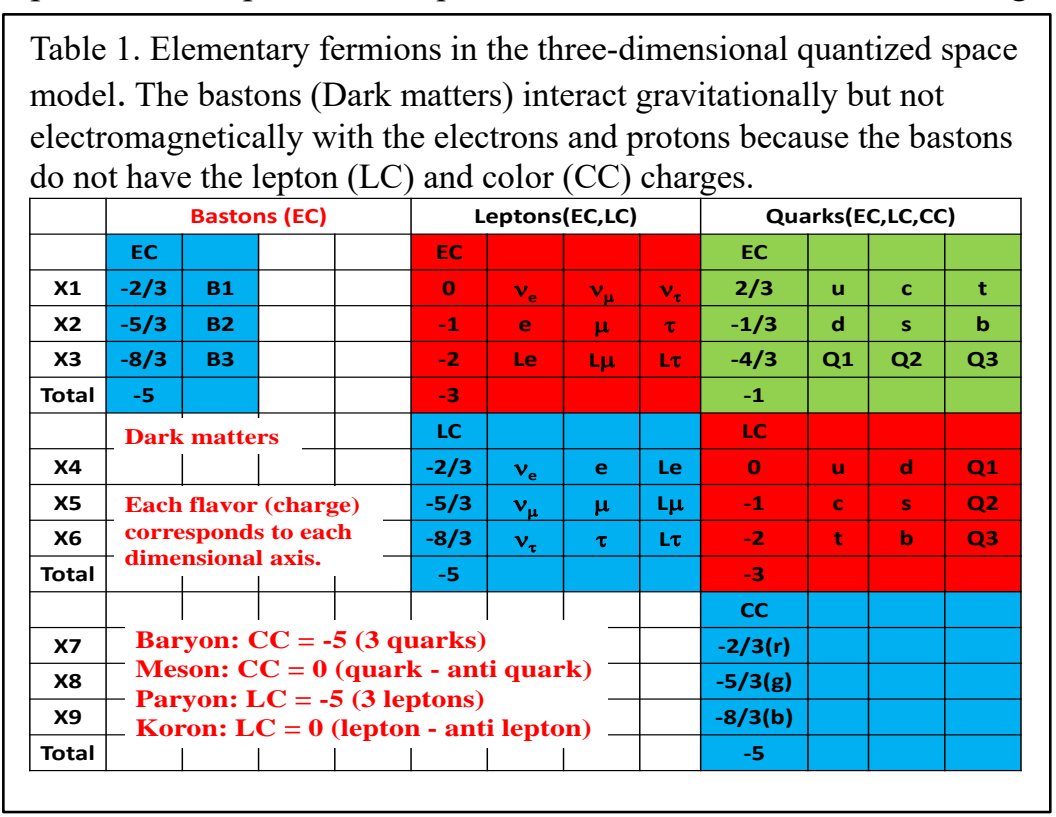

generations are called as the lepton charges (LC) or lepton flavors in the present work. The difference between the quarks and leptons is that the quarks have the three color flavors or three color charges (CC) of red (r), green (g) and blue (b) but the leptons do not. The quarks with the 
same charges of EC and LC are separated with three color charges (CC). Because the lepton charges and color charges have the three flavors, the electric charges are expected to have the three flavors. But the electric charges of the leptons and quarks have two flavors. The particles with the same lepton charges are separated with two electric charges of 0 and -1 for the leptons and $2 / 3$ and $-1 / 3$ for the quarks. The electric charges are quantized on the basis of the electron electric charge of -1 in Table 1 . Therefore, the heavy quarks with the electric charge of $-4 / 3$ and heavy leptons with the electric charge of -2 for the third missing electric charges are added to complete the three flavors of EC, LC and CC in the quarks and leptons in Table 1. The elementary fermions in Table 1 can be explained only by the three-dimensional quantized spaces [1,2]. Each flavor corresponds to each dimensional axis in Table. 1. This work needs the further researches

\begin{tabular}{|c|c|c|c|}
\hline SM & & $\begin{array}{l}\text { Leptons, Mesons, } \\
\text { Baryons (EC) }\end{array}$ & Quarks (EC,CC) \\
\hline $\begin{array}{l}\text { Long Range Force } \\
\text { (EM Force), Photon }\end{array}$ & & $F(E C)$, QED, $\gamma$ & \\
\hline Short Range Force & & $\begin{array}{l}\text { Weak Force } \\
\text { Massive bosons } \\
\left(\mathrm{Z}, \mathrm{W}^{+}, \mathrm{W}^{-}\right)(\mathrm{EC})\end{array}$ & $\begin{array}{l}\text { Strong Force, QCD, } \\
\text { Massless gluons } \\
\text { (CC) }\end{array}$ \\
\hline ESM (TQSM) & $\begin{array}{l}\text { Bastons (EC) } \\
\text { (Dark matters) }\end{array}$ & $\begin{array}{l}\text { Leptons, Mesons, } \\
\text { Baryons }(E C, L C))\end{array}$ & Quarks (EC,LC,CC) \\
\hline $\begin{array}{l}\text { Long Range Force } \\
\text { (EM Force), Photon } \\
\text { QED }\end{array}$ & $\begin{array}{l}F(E C) \\
\text { Dark photon } \\
\gamma(0)\end{array}$ & $\begin{array}{l}F(E C, L C)= \\
F(E C)+F(L C) \\
\text { Normal photon } \\
\gamma(0,0)\end{array}$ & $\begin{array}{l}F(E C, L C, C C)= \\
F(E C)+F(L C)+F(C C) \\
\text { Unobservable } \\
\text { photon } \\
\gamma(0,0,0)\end{array}$ \\
\hline $\begin{array}{l}\text { Short Range Force } \\
\text { Massive bosons }\end{array}$ & $\begin{array}{l}\text { Dark matter force } \\
\mathrm{Z} / \mathrm{W} / \mathrm{Y}(\mathrm{EC})\end{array}$ & $\begin{array}{l}\text { Weak force } \\
Z / W / Y(E C, L C)\end{array}$ & $\begin{array}{l}\text { Strong force } \\
Z / W / Y(E C, L C, C C)\end{array}$ \\
\hline \multicolumn{4}{|c|}{$\begin{array}{l}\text { SM: Standard Model, ESM: Extended Standard Model, } \\
\text { TQSM: Three-dimensional Quantized Space Model, QED: Quantum Eletro-Dynamics, } \\
\text { QCD: Quantum Chromo-Dynamics, EM Force: Electro-Magnetic Force } \\
\text { In SM, the force corresponding to the lepton charges (LC) is not considered. }\end{array}$} \\
\hline
\end{tabular}

on the new concepts. Only the electric charges have been quantized on the basis of the electron electric charge of -1. First, the new heavy leptons and new heavy quarks have the electric charges of -2 and $-4 / 3$, respectively in Table 1 . Then the sum of three electric charges is -3 for the leptons and -1 for the quarks. The summed electric charge $(-3)$ of the leptons is decreased by -2 when compared with the summed electric charge (-1) of the quarks. In Table 1, systematically three particles called as the bastons are expected. The sum of three electric charges is -5 for these new particles called as bastons. The electric charges for the bastons are $-2 / 3,-5 / 3$ and $-8 / 3$ which make the summed electric charge of -5 . The three-dimensional quantized spaces with the summed charges of $-5,-3$ and -1 are colored in blue, red and green, respectively in Table 1. Let's build up the three-dimensional quantized spaces of the leptons and quarks from the three-dimensional quantized space of the bastons. The three-dimensional quantized space of the bastons is in blue in Table 1. The leptons have the three-dimensional quantized spaces in red and in blue in Table 1. The quarks have the three-dimensional quantized spaces in green, in red and in blue in Table 1. Therefore, the leptons are made by adding the three-dimensional quantized space in red to the bastons. The lepton charges of the leptons are the same as the electric charges of the bastons. And the quarks are made by adding the three-dimensional quantized space in green to the leptons. The lepton charges of the quarks are the same as the electric charges of the leptons and the color 
charges of the quarks are the same as the lepton charges of the leptons and the electric charges of the bastons in Table 1. Therefore, all quantized charges of the elementary fermions can be assigned as shown in Table 1 . Then, the lepton charges are $0,-1$ and -2 for the quarks and $-2 / 3,-5 / 3$ and $8 / 3$ for the leptons. And the color charges for quarks are $-2 / 3,-5 / 3$ and $-8 / 3$. Therefore, the leptons and quarks should be described as (EC,LC) and (EC,LC,CC), respectively as shown in Table 1. Therefore, the bastons are described as (EC). These new particles have the properties the same as the dark matters have. These new particles interact gravitationally but not electromagnetically with the electrons, protons and quarks because the bastons do not have the lepton (LC) and color (CC) charges. Then, the bastons are the dark matters. Also, the leptons interact gravitationally but not electromagnetically with the quarks because the leptons do not have the color (CC) charges. The leptons can interact electromagnetically with the hadrons like protons because the hadrons of the mesons and baryons have the color charges of 0 and -, respectively [1,2]. It is called as the hadronization.

\section{3. $\quad 3.5 \mathrm{keV}, 18.7 \mathrm{keV}$ and $74.9 \mathrm{keV} \mathrm{x}$-rays and Q1 baryon atoms}

The heavy baryons including the Q1 heavy quark (Q1 baryons) can be made in the active galactic nucleus. This Q1 baryon can have the electric charges (EC) of -1 and -2. For example, the $\mathrm{Q}(\mathrm{Q} 1, \mathrm{~d}, \mathrm{~d})$ and $\mathrm{R}(\mathrm{Q} 1, \mathrm{~d}, \mathrm{u})$ baryons in Fig. 1 have the charge configurations of $(\mathrm{EC}, \mathrm{LC})=(-$ $2,0)$ and $(\mathrm{EC}, \mathrm{LC})=(-1,0)$, respectively. Then the protons with the charge configurations of

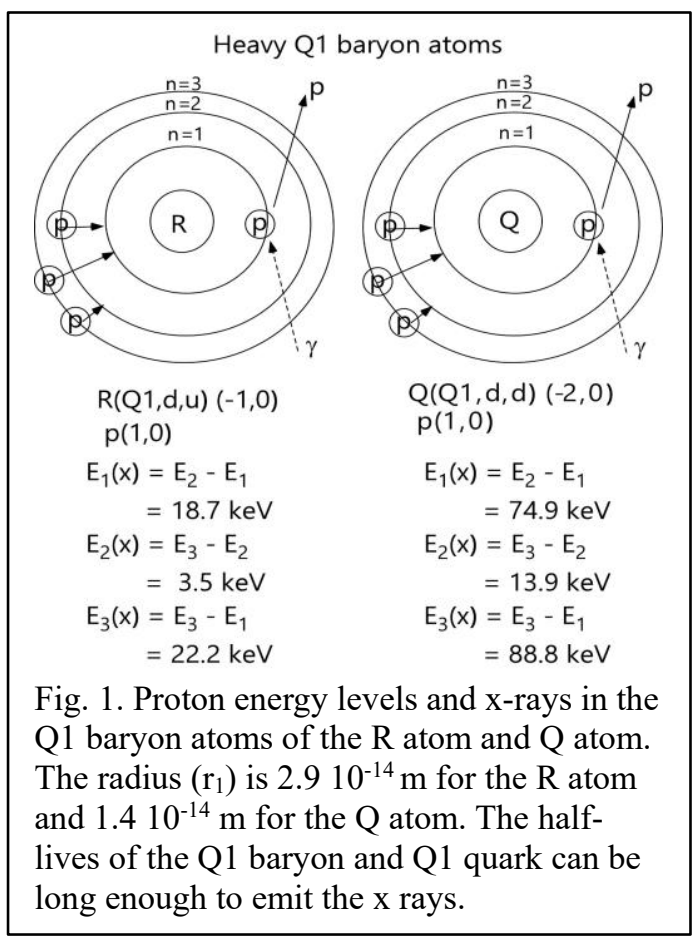
$(\mathrm{EC}, \mathrm{LC})=(1,0)$ can rotate around these $\mathrm{Q}$ and $\mathrm{R}$ baryons to form the Q1 baryon atoms. These Q1 baryon atoms are similar to the hydrogen atoms. The proton energy levels in the Q1 baryon atom are easily calculated in the same way as the electron energy levels in the hydrogen atom are calculated. The calculated x-ray energies are 18.7 $\mathrm{keV}$ and $3.5 \mathrm{keV}$ from the $\mathrm{R}$ atom and $74.9 \mathrm{keV}$ and $13.9 \mathrm{keV}$ from the $\mathrm{Q}$ atom in Fig. 1. The possible $18.7 \mathrm{keV}$ and $74.9 \mathrm{keV}$ x-ray peaks are found at the cosmic $x$-ray background spectra $[1,3,4]$. And the $74.9 \mathrm{keV} x$-ray peak is seen more clearly on the broadband energy spectrum of the X-ray pulsar 4U 0115+63 from IBIS/ISGRI and JEM-X(INTEGRAL) data in its bright state during the out-burst in May-June 2011 [1,5]. So it is thought that these $18.7 \mathrm{keV}$ and $74.9 \mathrm{keV} \mathrm{x}$-ray peaks are originated from the Q1 baryon atoms in Fig. 1. Also, the $3.5 \mathrm{keV}$ x-ray peak is expected from the Q1 baryon atom of the R atom in Fig. 1. And an emission line at $3.5 \mathrm{keV}$ was detected in the spectrum of the Cosmic X-ray Background using a total of 10 Ms Chandra observations towards the COSMOS Legacy and CDFS survey fields [6]. So it is thought that this observed 3.5 $\mathrm{keV}$-ray peak is originated from the $\mathrm{Q} 1$ baryon atom of the $\mathrm{R}$ atom but not from the pair annihilation of the dark matters. The presence of the $3.5 \mathrm{keV}$ cosmic $\mathrm{x}$-ray supports the presence of the Q1 quark with the electric charge of $-4 / / 3$ in Table 1. 


\section{Origins of high and ultra-high energy cosmic rays}

The dark matters can interact with the leptons as shown in Fig. 2. The B1-e and B1- $\mu$ interactions in Fig. 2 can enhance the numbers of the cosmic $v_{\mathrm{e}}$ and $v_{\mu}$ neutrinos when the electrons and muons get through the dense dark matter clouds near the galaxy center. Also, the B1 dark matters can be produced from the LHC accelerator. The observation of the enhanced $v_{\mathrm{e}}$ neutrinos will be the indirect evidence of the B1 dark matters produced from the LHC accelerator. The LHC and cosmic neutrino experiments will be interesting. And the rest mass energies of the leptons with the charge configuration of (EC,LC) and bastons (dark matters) with the charge configuration of (EC) are calculated by using the simple equations under the assumptions of E(B2) $=42.7 \mathrm{GeV}$ and $\mathrm{E}\left(v_{\tau}\right)=0.1 \mathrm{eV}$.

$\mathrm{F}(\mathrm{EC}, \mathrm{LC})=-23.24488+7.26341|\mathrm{EC}|-1.13858 \mathrm{EC}^{2}+0.62683|\mathrm{LC}|+0.22755 \mathrm{LC}^{2}$

$\mathrm{E}=8.136510^{38+2 \mathrm{~F}} \mathrm{eV}$ for leptons

$\mathrm{E}=17.150110^{38+2 \mathrm{~F}} \mathrm{eV}$ for bastons (dark matters)

Only the EC and LC charges of the leptons and bastons are used for the calculations of the rest masses. The rest masses of the leptons and bastons (dark matters) are compared with the experi-

Table 3. Rest masses of the leptons and bastons(dark matters) are
calculated and compared with the experimental values [1]. E= $\mathrm{mc}^{2}$.
\begin{tabular}{|c|c|c|c|c|c|}
\hline$(\mathrm{EC}, \mathrm{LC})$ & $\begin{array}{c}\mathrm{E}_{\text {exp }} \\
(\mathrm{eV})\end{array}$ & $\mathrm{E}_{\text {calc }}(\mathrm{eV})$ & $(\mathrm{EC}, \mathrm{LC})$ & $\mathrm{E}_{\exp }(\mathrm{eV})$ & $\mathrm{E}_{\text {calc }}(\mathrm{eV})$ \\
\hline$v_{\mathrm{e}}(0,-2 / 3)$ & $?$ & $2.87610^{-7}$ & $\mathrm{e}(-1,-2 / 3)$ & $5.1110^{5}$ & $5.1110^{5}$ \\
\hline$v_{\mu}(0,-5 / 3)$ & $?$ & $5.94710^{-5}$ & $\mu(-1,-5 / 3)$ & $1.05710^{8}$ & $1.05710^{8}$ \\
\hline$v_{\tau}(0,-8 / 3)$ & $?$ & $1.00010^{-1}$ & $\tau(-1,-8 / 3)$ & $1.77710^{9}$ & $1.77710^{9}$ \\
\hline $\mathrm{Le}(-2,-2 / 3)$ & $10^{12-14}$ & $2.53310^{13}$ & $\mathrm{~B} 1(-2 / 3)$ & $?$ & 26.121 \\
\hline $\mathrm{L} \mu(-2,-5 / 3)$ & $?$ & $5.23910^{15}$ & $\mathrm{~B} 2(-5 / 3)$ & $4.2710^{10}$ & $4.2710^{10}$ \\
\hline $\mathrm{L} \tau(-2,-8 / 3)$ & $?$ & $8.81110^{18}$ & $\mathrm{~B} 3(-8 / 3)$ & $?$ & $1.94810^{15}$ \\
\hline
\end{tabular}

mental values in Table 3. The rest mass energies of the leptons and dark matters are calculated in order to show the energy scales of these particles by using the simple equations. The parameter values are assigned by fitting the experimental rest mass energies. Then, the unknown neutrino masses and the masses of the heavy leptons ( $L e, L \mu, L \tau)$ can be calculated in Table 3 . And the masses of the B1 and B3 dark matters can be calculated, too. Also, it is assumed that the rest mass energies of Q1, Q2 and Q3 quarks correspond to the energies of the first knee, second knee and ankle parts of the ultra-high energy cosmic ray spectra, respectively [1,7]. And the color charge effects on the rest mass energies of the quarks are assumed to be negligibly small. Then $\mathrm{E}=10^{\mathrm{F}}$ $\mathrm{eV}$ and $\mathrm{F}(\mathrm{EC}, \mathrm{LC})=10.34076-16.01455|\mathrm{EC}|+15.02553 \mathrm{EC}^{2}+2.14|\mathrm{LC}|+0.005 \mathrm{LC}^{2}$ for Q1, Q2 and $\mathrm{Q} 3$ quarks. The obtained rest mass energies are $\mathrm{E}(\mathrm{Q} 1)=510^{15} \mathrm{eV}, \mathrm{E}(\mathrm{Q} 2)=710^{17} \mathrm{eV}$ and $\mathrm{E}(\mathrm{Q} 3)=10^{20} \mathrm{eV}$.

The 42.7(7) GeV peak was identified in the gamma-ray spectrum from the Fermi Large Area Telescope (LAT) in the directions of 16 massive nearby Galaxy Clusters [8]. The $42.7 \mathrm{GeV}$ peak is proposed as the B2 - anti B2 annihilation peak in Fig. 3. Then, the rest mass of the B2 dark matter particle is $42.7(7) \mathrm{GeV} / \mathrm{c}^{2}$. And the enhanced intensity was observed around $42.7 \mathrm{GeV}$ for the gamma ray spectra of supernova remnant (SNR), W44, as measured with the Fermi-LAT. This might be the B2 - anti B2 annihilation peak [9]. The calculated rest mass energy of the B1 dark matter is $26.121 \mathrm{eV}$. It will be interesting to look for the $26.121 \mathrm{eV}$ peak at the cosmic $\mathrm{x}$-ray. The calculated rest mass energies of three neutrinos are $2.87610^{-7} \mathrm{eV}$ for $v_{\mathrm{e}}, 5.94710^{-5} \mathrm{eV}$ for $\nu_{\mu}$ and $1.00010^{-1} \mathrm{eV}$ for $v_{\tau}$ in Table 3. It will be interesting to confirm these rest mass energies of three neutrinos in Table 3. The rest masses of the elementary fermions depend on both of EC and LC 
according to the above mass energy equations. The leptons are separated into three groups. First group is made of the three neutrinos with $\mathrm{EC}=0$ which have the low energy range. Second group is made of the electron, muon and tau lepton with $\mathrm{EC}=-1$ which have the high energy range. The third group is made of the $\mathrm{Le}, \mathrm{L} \mu$ and $\mathrm{L} \tau$ leptons with $\mathrm{EC}=-2$ which have the ultra-high energy range in Fig. 3. The $\mathrm{B} 1$ dark matter with $\mathrm{EC}=-2 / 3$ has the rest mass energy between the first group with $\mathrm{EC}=0$ and second group with $\mathrm{EC}=-1$. The $\mathrm{B} 2$ dark matter with $\mathrm{EC}=-5 / 3$ has the rest mass energy between the second group with $\mathrm{EC}=-1$ and third group with $\mathrm{EC}=-2$. The $\mathrm{B} 3$ dark matter with $\mathrm{EC}=-8 / 3$ has the rest mass energy similar to those of the third group with $\mathrm{EC}=-2$. The electron-positron annihilation peaks associated with the outburst of the micro-quasar V404 Cygni [10] were identified at the energy range of $410^{5}-210^{6} \mathrm{eV}$ consistent with the rest mass energy (5.11 $10^{5} \mathrm{eV}$ ) of the electron. The possible Le - anti Le annihilation peak was identified at the energy
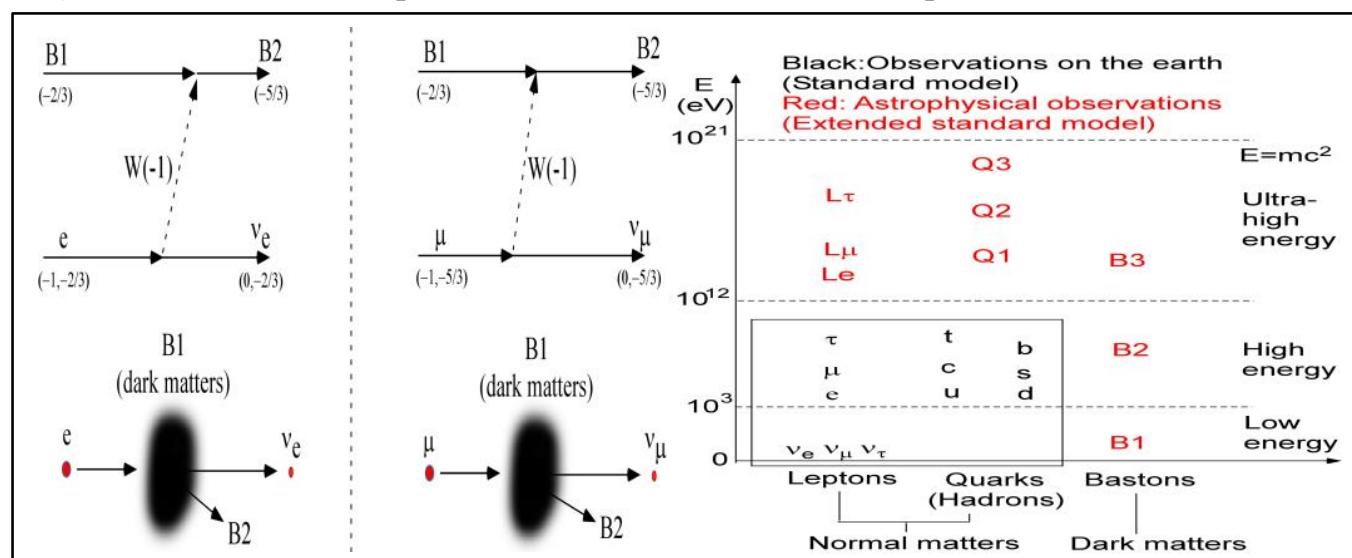

Fig. 2. B1 dark matter and lepton interactions to give the neutrino enhancement. New elementary fermion particles are compared. $\mathrm{E}=\mathrm{mc}^{2}$ is the rest mass energies of the particles.

range of $10^{12-14} \mathrm{eV}$ in the TeV gamma ray spectrum from RXJ1713.7-3946 with HESS and FermiLat data [11]. The calculated rest mass energy $\left(2.53410^{13} \mathrm{eV}\right)$ is consistent with the energy of the observed peak in Table 3. The high and ultra-high energy cosmic rays with the energy higher than $10^{9} \mathrm{eV}$ are originated from the decay and annihilations of the hadrons including the Q1, Q2 and Q3 quarks with the possible rest masses of $10^{15-20} \mathrm{eV} / \mathrm{c}^{2}$ and the heavy leptons as shown in Figs. 4 and $5[12,13]$.

For the cosmic gamma ray spectra in Fig. 3, the B2 - anti B2 annihilation peak is at 4.27 $10^{10} \mathrm{eV}$ and Le - anti Le annihilation peak is at $2.5310^{13} \mathrm{eV}$. And the smooth background curve of the cosmic gamma rays are caused by the gamma rays emitted from the Le decay. These shapes of the cosmic gamma ray spectra have been observed and reported in several references [11-14]. For the cosmic electrons, positrons and neutrinos, the cosmic ray spectra have the same origins in Fig. 3. These cosmic electron, positron and neutrino spectra are mainly originated from the Le decays [1,15-17]. It can be confirmed from the observed cosmic electron and positron spectra at the TeV energy range. And the cosmic electron, positron and neutrino spectra coming from the Q1 hadron decays are added around $510^{15} \mathrm{eV}$. The positron anomaly, e and $\mathrm{e}^{+}$spectra can be explained by the Le and anti Le decays [1,16-18]. The astrophysical neutrino spectra observed at the PeV energy range can be explained by using the Q1 hadron decays [1,15]. It is assumed that the decays of other heavy leptons and heavy hadrons have the relatively small contributions when compared with the Le decays. The ultra-high energy cosmic rays are mostly composed of the protons. The smooth background curve of the ultra-high energy cosmic ray spectra is caused by the gamma induced protons in Fig. 3 [7]. These protons are accelerated by the inelastic Compton scattering with the gamma rays emitted from the pair annihilations of Q1, Q2 and Q3 hadrons. And the first knee, second knee and ankle parts of the ultra-high energy cosmic ray spectra $[1,7]$ are explained by using the Q1-hadron, Q2-hadron and Q3-hadron decays in Fig. 3, respectively. The observed cosmic ray spectra can be found in Ref. [7,19]. 


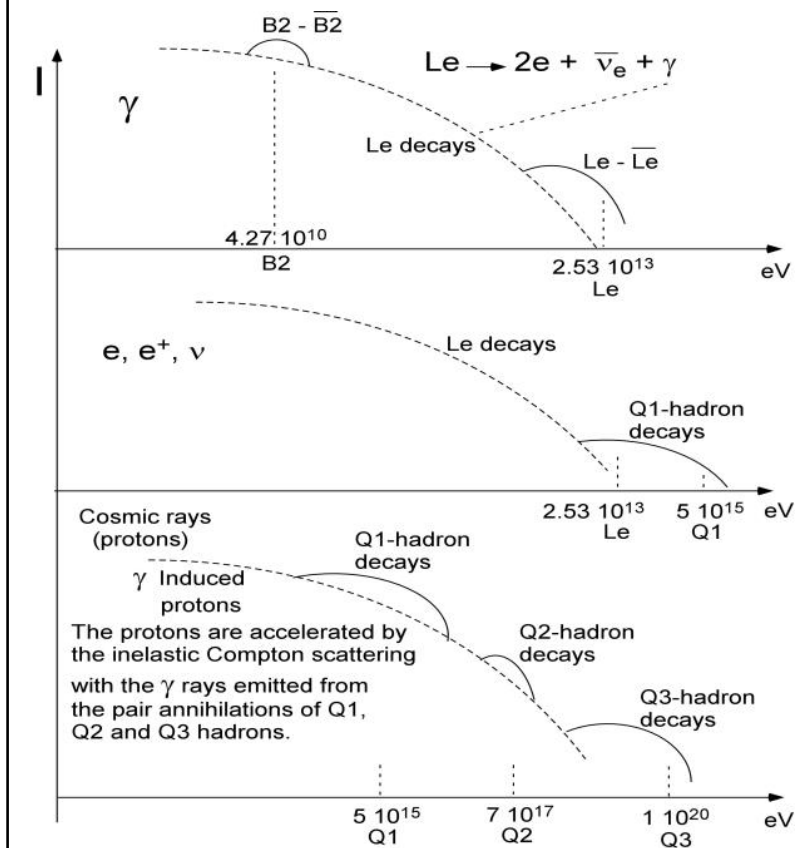

Fig. 3. Origins of the high and ultra-high energy cosmic rays and gamma rays.
The super-nova 1987A has been seen to have the three rings on the Hubble space telescope pictures. And the evidence of the neutron star is missing in the super- nova 1987A [20-22]. The neutrino masses of $21.4(12) \mathrm{eV} / \mathrm{c}^{2}$ and $4.0(5) \mathrm{eV} / \mathrm{c}^{2}$ are extracted from the antineutrino data from the super-nova 1987A [20]. These neutrino masses are too large. So, I tried to solve these two questions by using the B1 dark matter. Then, the new concept of the dark matter core collapse in addition to the normal matter core collapse is introduced in order to build the super-nova structure. The experimental anti-neutrino data are used to draw the conclusions in the present work. The more details on the experimental neutrino measurements can be found from the references $[20,21,22]$. The super-nova $1987 \mathrm{~A}$ antineutrino data can be drawn as shown in Fig. 4. The neutrino energy of $E(v)$ is related to the time of $t$. The equation of $2 \mathrm{E}^{2} \mathrm{t}=\mathrm{m}^{2} \mathrm{c}^{4} \mathrm{t}_{0}$ isused [20]. The curve $\mathrm{A}$ fits other data well except the 6 data. The curve A uses the proposed dark matter mass of B1. It is proposed that the B1 particles come from the SN 1987A to the earth. The B1 and anti $B 1$ dark matters coming from the SN1987A change the directions by the neutral boson $(Z(0))$ interactions with $p$ and $e$ in the earth atmosphere. The B1 and anti $B 1$ dark matters are pair-annihilated within the earth atmos-

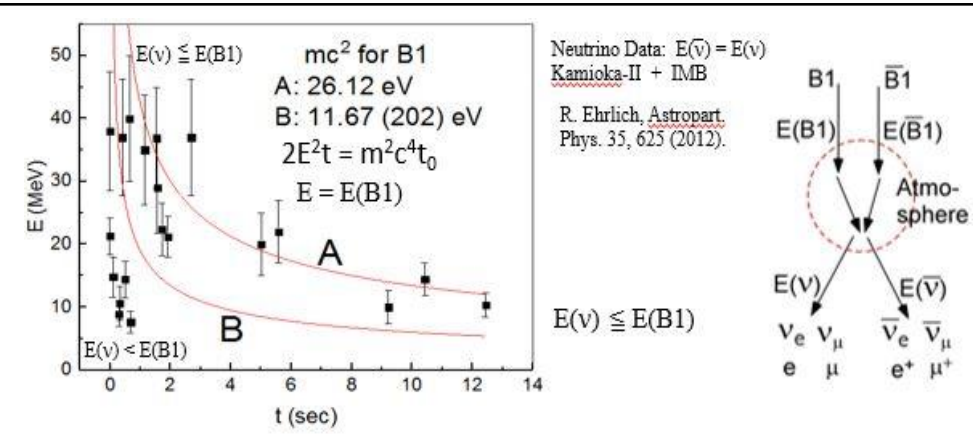

Fig. 4. Neutrino data of the super-nova 1987A. The B1 -anti B1 dark matter pair is annihilated to create the $v-$ anti $v$ pair.

phere and the $v$ and anti $v$ pair is created. These neutrinos are observed by using the detectors under the earth surface. The energies $\mathrm{E}(v)$ of the observed neutrinos are similar to the energies, $\mathrm{E}(\mathrm{B} 1)$ of the $\mathrm{B} 1$ dark matters. This supports indirectly that the rest mass energy of the B1 dark matter is $26.12 \mathrm{eV}$.

\section{Summary}

In summary, the three-dimensional quantized space model is introduced as the new extended standard model. Three generations of the leptons and quarks correspond to the lepton charges. Quarks have three charges of EC, LC and CC, and leptons have two charges of EC and LC. New particles of bastons with only one charge of EC are the dark matters. And the rest mass energies of the leptons with the charge configuration of (EC.LC) and bastons (dark matters) with the charge 
configuration of (EC) are calculated by using the simple equations. The standard model has the conservation rules of many quantum numbers such as baryon number, lepton number, B-L symmetry, hyper charge, weak charge, electric charge, color charge, quark flavor quantum number, lepton family number and $\mathrm{x}$-charge. However, the present model has only one conservation rule of EC, LC and CC charges. The calculated rest mass energies of three neutrinos are $2.87610^{-7} \mathrm{eV}$ for $\nu_{\mathrm{e}}, 5.94710^{-5} \mathrm{eV}$ for $\nu_{\mu}$ and $1.00010^{-1} \mathrm{eV}$ for $\nu_{\tau}$ in Table 3. And the calculated rest mass energy of the B1 dark matter is $26.121 \mathrm{eV}$ which is supported by the SN1987A data. It will be interesting to look for the $26.121 \mathrm{eV}$ peak at the cosmic $\mathrm{x}$-ray and at LHC. The ultra-high energy cosmic rays and gamma rays are originated from the decay and annihilations of the hadrons including the Q1, Q2 and Q3 quarks with the possible rest masses of $10^{15-20} \mathrm{eV} / \mathrm{c}^{2}$. It is thought that the $18.7 \mathrm{keV}, 3.5 \mathrm{keV}$ and $74.9 \mathrm{keV} \mathrm{x}$ ray peaks observed from the cosmic $\mathrm{x}$-ray background spectra are originated not from the pair annihilations of the dark matters but from the $\mathrm{x}$-ray emission of the Q1 baryon atoms. Also, see Refs. [23,24].

\section{References}

[1] Jae-Kwang Hwang, https://www.researchgate.net/publication/314246000 .

[2] Jae-Kwang Hwang, https://www.researchgate.net/publication/313247136.

[3] M. Turler et al., $A \& A 512$ (2010) A49.

[4] Yong Shi et al., The Astrophysical Journal 777 (2013) 6.

[5] P.A. Boldin et al., Astronomy Letters 39 (2013) 375.

[6] N. Cappelluti et al., arXiv: 1701.07932 (2017).

[7] S. Hussain, arXiv: 1301.6619v1 (2013).

[8] Y.F. Liang et al., Phys. Rev. D93 (2016) 103525.

[9] M. Ackermann et al., arXiv: 1302.3307v1 (2013).

[10] T. Siegert et al., arXiv: 1603.01169 (2016).

[11] V. Gammaldi, arXiv: 1412.7639 (2014).

[12] P. Meszaros et al., www2.astro.psu.edu/users/nnp/cr.html.

[13] J.N. Bahcall and E. Waxman, Phys. Lett. B556 (2003) 1.

[14] S. Federicici et al., arXiv: 1502.06355v1 (2015).

[15] M.G. Aartsen et al., arXiv: 1412.5106 (2014).

[16] L. Accardo et al., Phys. Rev. Lett. 113 (2014) 121101.

[17] M. Aguilar et al., Phys. Rev. Lett. 113 (2014) 121102.

[18] P. Lipari, arXiv: 1608.02018 (2016) and references there in.

[19] M.T. Dova, arXiv: 160407584v1 (2016).

[20] R. Ehrlich, Astropart. Phys. 35 (2012) 625.

[21] F. Vissani et al., arXiv: 1008.4726v1 (2010).

[22] G. Schatz, arXiv: 1507.07107v1 (2015).

[23] Jae-Kwang Hwang, Talk at Intl. Conf. on New trends in high energy physics, October 2-8 (2016).

[24] Jae-Kwang Hwang, Talk at American physical society April meeting 2017, January 28-31 (2017), https://absuploads.aps.org/presentation.cfm?pid=12521. 